\title{
Solvation Structures and Deactivation Pathways of Luminescent Isothiazole-Derived Nucleobases: tzA, tzG, and tzl
}

Adalberto Vasconcelos Sanches de Araújo1, Danillo Valverde ${ }^{2}$, Sylvio Canuto ${ }^{2}$ and Antonio Carlos Borin 1 ${ }^{1}$ Department of Fundamental Chemistry, Institute of Chemistry, University of São PauloAvenida Professor Lineu Prestes, 748, São Paulo, SP, 05508-000, Brazil

2Institute of Physics, University of São Paulo, Rua do Matão, 1371, São Paulo, SP, 05508-090, Brazil

\section{Optimized Geometries}

On the following pages, all the geometries used in this work. The structures are displayed the $\mathrm{XYZ}$ file format.

$\begin{array}{llll}14 & & \\ \text { tzA } & 1\left(\pi \pi^{*}\right) \min & \text { in gas phase } \\ \mathrm{N} & -2.365990 & -0.517520 & -0.318200 \\ \mathrm{C} & -2.237710 & -0.295840 & +0.975550 \\ \mathrm{~N} & -1.142610 & +0.037750 & +1.729870 \\ \mathrm{C} & +0.028430 & +0.161820 & +1.021600 \\ \mathrm{C} & +0.077030 & -0.049300 & -0.400650 \\ \mathrm{C} & -1.166070 & -0.384370 & -1.025180 \\ \mathrm{~N} & +1.255620 & +0.083530 & -1.056720 \\ \mathrm{~S} & +2.392900 & +0.491810 & +0.135250 \\ \mathrm{C} & +1.307360 & +0.486430 & +1.495630 \\ \mathrm{~N} & -1.226840 & -0.586750 & -2.325490 \\ \mathrm{H} & +1.620350 & +0.707400 & +2.515540 \\ \mathrm{H} & -0.376350 & -0.501750 & -2.878510 \\ \mathrm{H} & -2.120350 & -0.829330 & -2.746060 \\ \mathrm{H} & -3.161900 & -0.393020 & +1.559960\end{array}$

14

tzA ${ }^{1}$ (gs/mח*) CI in gas-phase

$\begin{array}{llll}\mathrm{N} & -2.234565 & -0.612620 & -0.228582 \\ \mathrm{C} & -2.116756 & -0.284343 & +1.007644 \\ \mathrm{~N} & -1.209943 & +0.781148 & +1.341532 \\ \mathrm{C} & +0.087822 & +0.324183 & +1.096837 \\ \mathrm{C} & +0.124517 & +0.084306 & -0.345643 \\ \mathrm{C} & -1.164128 & -0.103385 & -0.996792 \\ \mathrm{~N} & +1.306844 & +0.098818 & -0.917985 \\ \mathrm{~S} & +2.511107 & +0.191320 & +0.308278 \\ \mathrm{C} & +1.342187 & +0.390758 & +1.617516 \\ \mathrm{~N} & -1.193286 & -0.402659 & -2.310511 \\ \mathrm{H} & +1.638528 & +0.553401 & +2.647822 \\ \mathrm{H} & -0.370708 & -0.266330 & -2.872765 \\ \mathrm{H} & -2.040955 & -0.748303 & -2.724938 \\ \mathrm{H} & -2.596229 & -0.833103 & +1.816449\end{array}$

14

$\begin{array}{llll}\text { tzA }{ }^{1}\left(\pi \pi^{*}\right) t s & \text { gas phase } & \\ \mathrm{N} & -2.427698 & +0.110145 & +0.149607 \\ \mathrm{C} & -1.992818 & +1.364114 & +0.136256 \\ \mathrm{~N} & -0.885933 & +1.622664 & -0.643351 \\ \mathrm{C} & +0.282825 & +1.070464 & -0.123157 \\ \mathrm{C} & -0.010892 & -0.353965 & -0.027089 \\ \mathrm{C} & -1.429488 & -0.748679 & -0.149123 \\ \mathrm{~N} & +0.989412 & -1.160868 & +0.113449 \\ \mathrm{~S} & +2.423547 & -0.244522 & +0.228473 \\ \mathrm{C} & +1.605091 & +1.307192 & -0.019927 \\ \mathrm{~N} & -1.716696 & -2.056332 & -0.319931 \\ \mathrm{H} & -0.981417 & -2.721066 & -0.202985 \\ \mathrm{H} & -2.652674 & -2.349859 & -0.134429 \\ \mathrm{H} & -2.407979 & +2.097693 & +0.813931 \\ \mathrm{H} & +2.148500 & +2.233320 & -0.103981\end{array}$

14

tzA ${ }^{1}\left(\pi \pi^{*}\right)$ min in dioxane using ASEC-FEG

$\mathrm{N}+2.462300+0.000000-0.000000$

C $\quad+1.168370-0.636630+0.000720$

$\mathrm{N} \quad-0.000000+0.000000+0.000000$

C $\quad+0.082860+1.367460-0.028810$

C $\quad+1.330400+2.151200-0.082280$

C $\quad+2.523070+1.396620+0.028740$

$\mathrm{N} \quad+1.278480+3.492950-0.152130$

S $\quad-0.382980+3.898690-0.121710$

C $\quad-1.009940+2.302540-0.000000$

$\mathrm{N}+3.760750+1.926690+0.144440$

$\mathrm{H} \quad-2.074490+2.080370+0.090800$

$\mathrm{H} \quad+3.876930+2.936310+0.195070$

$\mathrm{H} \quad+4.566170+1.307640+0.218160$

$\mathrm{H} \quad+1.195690-1.728730+0.000610$ 
14

tzA ${ }^{1}\left(\pi \pi^{*}\right) \mathrm{min}$ in water using ASEC-FEG

$\begin{array}{llll}\mathrm{N} & +2.478900 & +0.000000 & +0.000000 \\ \mathrm{C} & +1.230920 & -0.580420 & -0.001600 \\ \mathrm{~N} & +0.000000 & +0.000000 & +0.000000 \\ \mathrm{C} & +0.055050 & +1.380230 & +0.006870 \\ \mathrm{C} & +1.310020 & +2.158160 & +0.021210 \\ \mathrm{C} & +2.543090 & +1.398380 & +0.014490 \\ \mathrm{~N} & +1.194530 & +3.513700 & +0.030540 \\ \mathrm{~S} & -0.494880 & +3.870460 & +0.008970 \\ \mathrm{C} & -1.058530 & +2.240770 & +0.000000 \\ \mathrm{~N} & +3.764880 & +1.921410 & +0.014280 \\ \mathrm{H} & -2.116420 & +1.975090 & -0.007970 \\ \mathrm{H} & +3.916330 & +2.946840 & +0.036060 \\ \mathrm{H} & +4.584870 & +1.277780 & -0.005260 \\ \mathrm{H} & +1.243550 & -1.674890 & -0.005630\end{array}$

15

tzG ${ }^{1}\left(\pi \pi^{*}\right) \min$ gas phase

N $-2.001770+0.490160$

C $-1.951910-0.847050$

N $-0.803490-1.580590$

C $+0.346160-0.845820$

C $+0.399520+0.635080$

C $-0.822130+1.385010$

$\mathrm{N}+1.616590+1.205050$

$\mathrm{S}+2.793620-0.099920$

C $+1.636190-1.382690$

$\mathrm{N} \quad-3.088930-1.573810$

O $-1.057500+2.595530$

$\mathrm{H} \quad-2.886170+0.989250$

$\mathrm{H} \quad+1.898680-2.440160$

$\mathrm{H} \quad-3.994680-1.147200$

H $\quad-2.983030-2.566530$

$-0.106640$

$-0.059300$

$+0.013440$

$+0.028820$

$-0.004300$

$-0.069080$

$+0.050390$

$+0.123170$

$+0.098200$

$-0.122820$

$-0.101810$

$-0.189780$

$+0.156920$

$+0.038970$

$+0.060870$
15

tzG ${ }^{1}$ (gs/mח*) CI in gas-phase

$\begin{array}{llll}\text { N } & -2.270468 & -0.313109 & -0.423717 \\ \mathrm{C} & -2.281457 & -0.129674 & +0.961748 \\ \mathrm{~N} & -1.380825 & +0.832870 & +1.357821 \\ \mathrm{C} & -0.108215 & +0.450565 & +1.059889 \\ \mathrm{C} & +0.103860 & -0.381180 & -0.123454 \\ \mathrm{C} & -1.053740 & -0.717400 & -1.003187 \\ \mathrm{~N} & +1.330978 & -0.665851 & -0.422536 \\ \mathrm{~S} & +2.371459 & +0.037610 & +0.705078 \\ \mathrm{C} & +1.122402 & +0.802567 & +1.610193 \\ \mathrm{~N} & -2.209435 & -1.332497 & +1.723989 \\ \mathrm{O} & -0.978743 & -1.268932 & -2.064518 \\ \mathrm{H} & +1.285603 & +1.425926 & +2.467508 \\ \mathrm{H} & -3.097908 & -0.632492 & -0.914718 \\ \mathrm{H} & -2.832437 & -2.052884 & +1.360883 \\ \mathrm{H} & -2.470097 & -1.194514 & +2.697525\end{array}$

15

tzG ${ }^{1}\left(\pi \pi^{*}\right)$ ts gas phase

$\begin{array}{llll}\mathrm{C} & -1.645920 & -0.527672 & -0.004069 \\ \mathrm{C} & +0.613059 & -0.511234 & -0.052587 \\ \mathrm{C} & +0.640730 & +0.935937 & -0.112420 \\ \mathrm{C} & -0.570511 & +1.671579 & -0.083759 \\ \mathrm{~S} & +3.073765 & +0.226339 & -0.088972 \\ \mathrm{O} & -0.769557 & +2.863700 & -0.047774 \\ \mathrm{~N} & -1.711689 & +0.807343 & -0.115848 \\ \mathrm{~N} & -0.522318 & -1.220012 & +0.051482 \\ \mathrm{~N} & +1.903702 & +1.464894 & +0.047651 \\ \mathrm{C} & +1.914310 & -1.070670 & +0.007509 \\ \mathrm{~N} & -2.814918 & -1.211625 & +0.094436 \\ \mathrm{H} & -2.594385 & +1.274050 & -0.059223 \\ \mathrm{H} & +2.147176 & -2.058574 & +0.369378 \\ \mathrm{H} & -3.637308 & -0.806755 & -0.302171 \\ \mathrm{H} & -2.725317 & -2.200508 & -0.021143\end{array}$

15

tzG ${ }^{1}\left(\pi \pi^{*}\right) \mathrm{min}$ in dioxane using ASEC-FEG

$\mathrm{N}+2.331550+1.407370+0.065650$

$\mathrm{C}+2.301340+0.000000-0.000000$

$\mathrm{N}+1.154290-0.711780-0.008600$

C $+0.000000+0.000000+0.000000$

C $-0.060680+1.489020-0.000000$

C $+1.149890+2.254060+0.039190$

$\mathrm{N} \quad-1.285630+2.061160-0.024700$

S $\quad-2.429470+0.802760 \quad-0.038980$

C $\quad-1.315160 \quad-0.519470 \quad-0.010710$

$\mathrm{N}+3.499410-0.646800-0.023620$

$0+1.331460+3.489270+0.056770$

$\mathrm{H}+3.228860+1.910050+0.109380$

H $\quad-1.633220-1.563940-0.016800$

$\mathrm{H} \quad+4.385880-0.151240-0.067390$

$\mathrm{H} \quad+3.488310-1.666750 \quad-0.070590$

15

tzG ${ }^{1}\left(\pi \pi^{*}\right) \mathrm{min}$ in water using ASEC-FEG

$\mathrm{N}+2.343910+1.381710+0.180950$

$\mathrm{C}+2.329410-0.000000+0.000000$

$\mathrm{N}+1.194560 \quad-0.708020-0.034430$

C $-0.000000+0.000000+0.000000$

C $-0.116950+1.444920+0.000000$

C $+1.079280+2.213210+0.081840$

N $-1.390390+1.991990-0.089480$

S $\quad-2.480560+0.652940 \quad-0.145870$

C $\quad-1.269180-0.591050 \quad-0.062750$

$\mathrm{N}+3.565610 \quad-0.636880-0.103650$

$0+1.276460+3.466680+0.061850$

$\mathrm{H}+3.230010+1.890490+0.440700$

$\mathrm{H} \quad-1.510750-1.654570-0.100260$

$\mathrm{H} \quad+4.442430-0.115320-0.371050$

$\mathrm{H} \quad+3.524710 \quad-1.683840 \quad-0.235140$ 
13

$\begin{array}{llll}\text { tzI }{ }^{1}\left(\pi \pi^{*}\right) \mathrm{min} & \text { gas phase } \\ \mathrm{N} & -2.328280 & -0.157870 & -0.000020 \\ \mathrm{C} & -1.994800 & -1.440160 & -0.000020 \\ \mathrm{~N} & -0.731210 & -1.923850 & -0.000030 \\ \mathrm{C} & +0.241800 & -0.975300 & -0.000030 \\ \mathrm{C} & -0.007060 & +0.492190 & -0.000040 \\ \mathrm{C} & -1.351020 & +0.976480 & -0.000050 \\ \mathrm{~N} & +1.067820 & +1.290730 & +0.000100 \\ \mathrm{~S} & +2.484230 & +0.249640 & +0.000070 \\ \mathrm{C} & +1.611310 & -1.236980 & +0.000040 \\ \mathrm{O} & -1.854790 & +2.099860 & -0.000110 \\ \mathrm{H} & -3.306410 & +0.135740 & -0.000030 \\ \mathrm{H} & +2.082180 & -2.221660 & -0.000010 \\ \mathrm{H} & -2.812100 & -2.171510 & -0.000030\end{array}$

13

$\operatorname{tzI~}^{1}\left(\mathrm{gs} / \pi \pi^{*}\right) \mathrm{CI}$ in gas phase

$\begin{array}{llll}\mathrm{N} & -2.275340 & -0.423919 & -0.321085 \\ \mathrm{C} & -2.102330 & -0.364584 & +1.070472 \\ \mathrm{~N} & -1.266564 & +0.657836 & +1.437870 \\ \mathrm{C} & +0.040029 & +0.374432 & +1.076276 \\ \mathrm{C} & +0.160093 & -0.250639 & -0.235173 \\ \mathrm{C} & -1.075010 & -0.579753 & -1.047075 \\ \mathrm{~N} & +1.373951 & -0.410353 & -0.706776 \\ \mathrm{~S} & +2.481600 & +0.154890 & +0.423230 \\ \mathrm{C} & +1.288490 & +0.685465 & +1.561704 \\ \mathrm{O} & -1.059126 & -0.957263 & -2.192020 \\ \mathrm{H} & +1.571238 & +1.158558 & +2.486127 \\ \mathrm{H} & -2.104258 & -1.293975 & +1.653384 \\ \mathrm{H} & -3.057974 & -0.918823 & -0.731690\end{array}$

13

tzI ${ }^{1}\left(\pi \pi^{*}\right)$ ts gas phase

C $\quad-1.936866 \quad-1.425788 \quad-0.002859$

C $\quad+0.256077-0.896084+0.005116$

C $-0.041813+0.470284+0.004130$

$\begin{array}{llll}\text { C } & -1.396391 & +0.962634 & -0.003373\end{array}$

$\mathrm{s}+2.621139+0.307855+0.000932$

o $\quad-1.754633+2.112039-0.009155$

N $\quad-2.295148 \quad-0.099626 \quad-0.002026$

N $\quad-0.735968-1.869753+0.001513$

$\mathrm{N}+1.132657+1.179140+0.009950$

C $+1.633446-1.166993+0.009485$

H $\quad-3.263673+0.146678-0.013793$

$\mathrm{H} \quad+2.106167-2.134094+0.006173$

$\begin{array}{llll}\text { H } & -2.762071 & -2.124388 & -0.006117\end{array}$

13

tzI ${ }^{1}\left(\pi \pi^{*}\right) \mathrm{min}$ in dioxane using ASEC-FEG

$\mathrm{N}+2.326860+1.390970-0.011130$

C $+2.293220-0.000000+0.000000$

$\mathrm{N}+1.080270 \quad-0.752040-0.001350$

C $+0.000000+0.000000+0.000000$

C $-0.060940+1.491480-0.000000$

C $+1.179410+2.250090-0.008120$

$\mathrm{N}-1.272950+2.080070+0.003730$

S $-2.436320+0.843980+0.007190$

C $-1.369830 \quad-0.464710+0.003020$

O $+1.367290+3.463890-0.013440$

$\mathrm{H}+3.226940+1.879710-0.023110$

$\mathrm{H} \quad-1.710180-1.505620+0.001160$

$\mathrm{H}+3.253080-0.517140+0.005890$
13

$\begin{array}{llll}\mathrm{tzI}{ }^{1}\left(\pi \pi^{*}\right) \mathrm{min} & \text { water using ASEC-FEG } \\ \mathrm{N} & +2.328470 & +1.405810 & +0.015020 \\ \mathrm{C} & +2.277710 & -0.000000 & -0.000000 \\ \mathrm{~N} & +1.102240 & -0.740400 & -0.005110 \\ \mathrm{C} & +0.000000 & +0.000000 & +0.000000 \\ \mathrm{C} & -0.042800 & +1.503870 & +0.000000 \\ \mathrm{C} & +1.196980 & +2.264150 & +0.008200 \\ \mathrm{~N} & -1.272600 & +2.086460 & -0.006100 \\ \mathrm{~S} & -2.420970 & +0.849050 & +0.001450 \\ \mathrm{C} & -1.350370 & -0.467890 & +0.003820 \\ \mathrm{O} & +1.344740 & +3.501820 & +0.009660 \\ \mathrm{H} & +3.241050 & +1.869530 & +0.031570 \\ \mathrm{H} & -1.700150 & -1.508380 & +0.010230 \\ \mathrm{H} & +3.242480 & -0.510390 & -0.004440\end{array}$




\section{MEP Structures}

14

tzA mep:0

N -1.030748

C +1.407868

$\mathrm{N}+1.343155$

N -1.142638

C -2.093429

C +0.110648

C +0.150231

C -1.091830

$\mathrm{S}+2.464350$

H +1.724899

H -0.259250

H -1.967824

H -3.035606
$-0.452765$

$+0.061493$

$+0.495009$

$+0.152181$

$-0.437776$

$-0.250838$

$+0.187656$

$+0.012511$

$-0.314924$

$+0.518863$

$+0.690095$

$-0.591435$

$-0.893745$

$-0.371613$
14

tzA mep:1

$\mathrm{N} \quad-2.212984$

N -1.008836

C +1.400666

N +1.334377

N -1.152895

C -2.107222

C +0.107150

C $\quad+0.128677$

C $\quad-1.077037$

S $\quad+2.480172$

$\mathrm{H} \quad+1.720252$

H $\quad-0.294321$

H -1.955643

H $\quad-3.023166$
$-0.453898$

$+0.064272$

$+0.496563$

$+0.151182$

$-0.438445$

$-0.255633$

$+0.190634$

$+0.004842$

$-0.311608$

$+0.521343$

$+0.689253$

$-0.599868$

$-0.911360$

$-0.368595$
14

tzA mep: 2

N $\quad-2.234308$

N -1.008494

C +1.398522

$\mathrm{N}+1.322588$

N -1.173395

C $\quad-2.114867$

C $\quad+0.118807$

C +0.126725

C -1.077940

S +2.505692

$\mathrm{H} \quad+1.711258$

H $\quad-0.320661$

H $\quad-1.975278$

H -3.024300
$-0.456016$

$+0.062431$

$+0.498288$

$+0.149545$

$-0.442018$

$-0.260641$

$+0.192936$

$+0.004055$

$-0.311195$

$+0.526359$

$+0.689882$

$-0.602430$

$-0.941206$

$-0.370787$
$-0.265004$

$+1.821480$

$+1.536068$

$-0.985887$

$-2.346299$

$+1.086800$

$+1.070936$

$-0.347917$

$-0.990221$

$+0.202293$

$+2.562274$

$-2.821515$

$-2.722546$

$+1.634932$
$-0.263227$

$+1.811677$

$+1.558248$

$-0.977801$

$-2.349184$

$+1.071731$

$+1.102767$

$-0.367188$

$-0.996343$

$+2.565749$

$-2.831124$

$-2.705634$

$+1.630834$
$+0.197996$

14

tzA mep: 3

$\begin{array}{llll}\mathrm{N} & -2.254779 & -0.457535 & -0.266100 \\ \mathrm{~N} & -1.017258 & +0.058673 & +1.793638 \\ \mathrm{C} & +1.392032 & +0.496624 & +1.569445 \\ \mathrm{~N} & +1.319032 & +0.148887 & -0.952139 \\ \mathrm{~N} & -1.181577 & -0.452952 & -2.320301 \\ \mathrm{C} & -2.128244 & -0.267215 & +1.019655 \\ \mathrm{C} & +0.134484 & +0.194753 & +1.101827 \\ \mathrm{C} & +0.137134 & +0.007728 & -0.343838 \\ \mathrm{C} & -1.072314 & -0.306902 & -0.996463 \\ \mathrm{~S} & +2.522019 & +0.529428 & +0.191037 \\ \mathrm{H} & +1.702715 & +0.685677 & +2.580764 \\ \mathrm{H} & -0.343783 & -0.543823 & -2.855806 \\ \mathrm{H} & -2.008666 & -0.897872 & -2.658896 \\ \mathrm{H} & -3.031394 & -0.377370 & +1.603830\end{array}$

14

tzA mep: 4

$\begin{array}{llll}\mathrm{N} & -2.267681 & -0.456266 & -0.267502 \\ \mathrm{~N} & -1.030167 & +0.053484 & +1.781615 \\ \mathrm{C} & +1.389115 & +0.494189 & +1.568303 \\ \mathrm{~N} & +1.320533 & +0.152201 & -0.952994 \\ \mathrm{~N} & -1.169447 & -0.471201 & -2.289463 \\ \mathrm{C} & -2.148141 & -0.272468 & +1.006753 \\ \mathrm{C} & +0.140701 & +0.195490 & +1.099544 \\ \mathrm{C} & +0.147671 & +0.013157 & -0.334534 \\ \mathrm{C} & -1.061446 & -0.300580 & -0.984440 \\ \mathrm{~S} & +2.528453 & +0.530623 & +0.181842 \\ \mathrm{H} & +1.705563 & +0.679782 & +2.579155 \\ \mathrm{H} & -0.354615 & -0.445949 & -2.866171 \\ \mathrm{H} & -2.041574 & -0.780982 & -2.662796 \\ \mathrm{H} & -3.050136 & -0.384784 & +1.594211\end{array}$

14

tzA mep: 5

N -1.032145

C +1.391675

$\mathrm{N}+1.317681$

$-1.158959$

$-2.153690$

$+0.142109$

$+0.148152$

$-1.060502$

$+2.527999$

$+1.709388$

$-0.363431$

$-2.062435$

$-0.445554$

$-0.269768$

$+0.045807$

$+0.488666$

$+0.161388$

$-0.499492$

$-0.265128$

$+0.189426$

$+0.013439$

$-0.302175$

$+0.532663$

$+0.674966$

$-0.363833$

$+1.780864$

$+1.567545$

$-0.954999$

$-2.281094$

$+1.004496$

$+1.101178$

$-0.331840$

$-0.980197$

$+0.178821$

$+2.578182$

$-2.841564$

$-2.672797$

$+1.620355$
$-2.869527$

$-2.677560$

$+1.590370$ 
14

tzA mep: 6

$\begin{array}{llll}\mathrm{N} & -2.272578 & -0.438629 & -0.270162 \\ \mathrm{~N} & -1.029244 & +0.039772 & +1.782517 \\ \mathrm{C} & +1.392338 & +0.482655 & +1.568472 \\ \mathrm{~N} & +1.316082 & +0.165751 & -0.955668 \\ \mathrm{~N} & -1.158886 & -0.504714 & -2.282004 \\ \mathrm{C} & -2.152564 & -0.260467 & +1.004603 \\ \mathrm{C} & +0.142489 & +0.187014 & +1.101326 \\ \mathrm{C} & +0.146785 & +0.015012 & -0.332969 \\ \mathrm{C} & -1.060828 & -0.303912 & -0.981589 \\ \mathrm{~S} & +2.526843 & +0.535424 & +0.179562 \\ \mathrm{H} & +1.711055 & +0.660308 & +2.580416 \\ \mathrm{H} & -0.357613 & -0.398750 & -2.868695 \\ \mathrm{H} & -2.058264 & -0.682649 & -2.676312 \\ \mathrm{H} & -3.058589 & -0.354606 & +1.589647\end{array}$

14

tzA mep: 7

N -2.270596

N -1.033529

C +1.391181

$\mathrm{N}+1.318096$

N -1.161537

C -2.153096

C +0.142086

C +0.148892

C -1.059854

S +2.527529

H $\quad+1.707739$

H -0.356621

H $\quad-2.052918$

H -3.057482
$-0.453369$

$+0.052528$

$+0.490213$

$+0.156068$

$-0.481922$

$-0.267343$

$+0.189881$

$+0.007925$

$-0.304378$

$+0.533338$

$+0.685044$

$-0.396339$

$-0.703995$

$-0.370533$
$-0.268814$

$+1.780154$

$+1.567625$

$-0.954338$

$-2.282830$

$+1.004571$

$+1.101431$

$-0.330769$

$-0.980067$

$+0.178796$

$+2.577125$

$-2.867686$

$-2.672442$

$+1.590759$
14

tzA mep: 8

$\begin{array}{ll}\mathrm{N} & -2.270783 \\ \mathrm{~N} & -1.033296 \\ \mathrm{C} & +1.391599 \\ \mathrm{~N} & +1.318317 \\ \mathrm{~N} & -1.161127 \\ \mathrm{C} & -2.152749 \\ \mathrm{C} & +0.142118 \\ \mathrm{C} & +0.148663 \\ \mathrm{C} & -1.060331 \\ \mathrm{~S} & +2.527967 \\ \mathrm{H} & +1.706072 \\ \mathrm{H} & -0.358788 \\ \mathrm{H} & -2.056882 \\ \mathrm{H} & -3.056346\end{array}$

$-0.451315$

$+0.051955$

$+0.488093$

$+0.157135$

$-0.486804$

$-0.267998$

$+0.189176$

$+0.009388$

$-0.301867$

$+0.534066$

$+0.694339$

$-0.385814$

$-0.682094$

$-0.375069$
14

tzA mep: 9

$\begin{array}{llll}\mathrm{N} & -2.270782 & -0.451315 & -0.269002 \\ \mathrm{~N} & -1.033295 & +0.051955 & +1.780561 \\ \mathrm{C} & +1.391596 & +0.488093 & +1.567771 \\ \mathrm{~N} & +1.318318 & +0.157134 & -0.954122 \\ \mathrm{~N} & -1.161124 & -0.486804 & -2.282706 \\ \mathrm{C} & -2.152748 & -0.267993 & +1.004696 \\ \mathrm{C} & +0.142116 & +0.189175 & +1.101573 \\ \mathrm{C} & +0.148667 & +0.009386 & -0.331330 \\ \mathrm{C} & -1.060335 & -0.301863 & -0.980908 \\ \mathrm{~S} & +2.527963 & +0.534069 & +0.179254 \\ \mathrm{H} & +1.706070 & +0.694335 & +2.575713 \\ \mathrm{H} & -0.358787 & -0.385817 & -2.868239 \\ \mathrm{H} & -2.056884 & -0.682093 & -2.676013 \\ \mathrm{H} & -3.056341 & -0.375071 & +1.591614\end{array}$

15

tzG mep: 0

C -1.638610

$-0.576111$

$-0.108831$

C +0.614199

$-0.522509$

$+0.019306$

C +0.666788

C $\quad-0.582392$

$+0.906869$

$+1.682515$

$+3.013391$

$-0.726306$

$-1.689907$

$+0.277741$

$+2.893742$

$+0.807806$

$-0.545772$

$-1.285682$

$+1.877720+1.504327$

$+1.919164$

$-1.032581$

$-2.871608$

$-1.205396$

$-2.584675+1.266950$

$+2.222630$

$-2.080621$

$-3.604153$

$-0.799537$

$-2.200720$

$+0.007061$

$-0.090080$

$+0.143429$

$-0.139752$

$-0.139749$

$-0.019753$

$+0.066485$

$+0.097956$

$-0.250874$

$-0.304497$

$+0.127088$

$+0.330586$

$-0.055885$

15

tzG mep: 1

$\begin{array}{llll}\mathrm{C} & -1.651622 & -0.552428 & -0.113137 \\ \mathrm{C} & +0.607485 & -0.534802 & +0.020285 \\ \mathrm{C} & +0.636797 & +0.928371 & +0.004076 \\ \mathrm{C} & -0.560745 & +1.675363 & -0.089127 \\ \mathrm{~S} & +3.022824 & +0.276513 & +0.143791 \\ \mathrm{O} & -0.734541 & +2.877793 & -0.139327 \\ \mathrm{~N} & -1.699873 & +0.796690 & -0.139057 \\ \mathrm{~N} & -0.536242 & -1.265585 & -0.022557 \\ \mathrm{~N} & +1.877339 & +1.510863 & +0.066570 \\ \mathrm{C} & +1.913741 & -1.053459 & +0.098326 \\ \mathrm{~N} & -2.853071 & -1.204445 & -0.240128 \\ \mathrm{H} & -2.575934 & +1.246717 & -0.301240 \\ \mathrm{H} & +2.215907 & -2.081557 & +0.126844 \\ \mathrm{H} & -3.612966 & -0.792883 & +0.263354 \\ \mathrm{H} & -2.772932 & -2.186661 & -0.069871\end{array}$


15

tzG mep: 2

$\begin{array}{llll}\mathrm{C} & -1.662696 & -0.529304 & -0.117089 \\ \mathrm{C} & +0.612539 & -0.530993 & +0.021071 \\ \mathrm{C} & +0.633590 & +0.932694 & +0.002123 \\ \mathrm{C} & -0.552368 & +1.669650 & -0.089739 \\ \mathrm{~S} & +3.036734 & +0.269871 & +0.144820 \\ \mathrm{O} & -0.755879 & +2.864271 & -0.139734 \\ \mathrm{~N} & -1.712874 & +0.796693 & -0.137547 \\ \mathrm{~N} & -0.535113 & -1.244631 & -0.029575 \\ \mathrm{~N} & +1.872011 & +1.511665 & +0.066378 \\ \mathrm{C} & +1.901088 & -1.064080 & +0.099209 \\ \mathrm{~N} & -2.827433 & -1.208272 & -0.225582 \\ \mathrm{H} & -2.587302 & +1.262341 & -0.289310 \\ \mathrm{H} & +2.191670 & -2.100574 & +0.126498 \\ \mathrm{H} & -3.654862 & -0.776893 & +0.136272 \\ \mathrm{H} & -2.751459 & -2.192236 & -0.054863\end{array}$

15

tzG mep:3

C -1.670916

C +0.615354

$-0.515703$

$-0.519760$

C +0.638935

$+0.940576$

$-0.553001$

$+3.049184$

$-0.787117$

$-1.723662$

$-0.534270$

$+1.878813$

$+1.892349$

$-2.806141$

$-2.594435$

$+2.162472$

$-3.688792$

H $\quad-2.730968$
$+1.674496$

$+0.255050$

$+2.863656$

$+0.804026$

$-1.229400$

$+1.505602$

$-1.064836$

$-1.221016$

$+1.282530$

$-2.105222$

$-0.789756$

$-2.204571$
$-0.118829$

$+0.020055$

$-0.001301$

$-0.092321$

$+0.146850$

$-0.140428$

$-0.135530$

$-0.035806$

$+0.065638$

$+0.101454$

$-0.209476$

$-0.253334$

$+0.129567$

$-0.036246$

$-0.053148$
15

tzG mep: 4

C $\quad-3.160904$

C +1.162135

C +1.213274

C -1.050093

S +5.772787

$-1.528491$

$-3.263939$

$-1.004371$

$+3.568770$

$+3.561160$

$-5.277754$

$-4.914478$

$+4.047064$

$-6.988117$

$-5.122096$
$-0.968411$

$-0.962860$

$+1.797869$

$+3.180477$

$+0.458264$

$+5.421498$

$+1.522733$

$-2.308444$

$+2.840208$

$-2.011238$

$-2.328005$

$+2.432751$

$-3.983823$

$-1.540175$

$-4.201617$
$-0.227613$

$+0.047925$

$-0.018791$

$-0.167626$

$+0.279467$

$-0.239091$

$-0.256935$

$-0.062038$

$+0.081744$

$+0.219578$

$-0.386680$

$-0.416485$

$+0.287725$

$-0.350650$

$-0.232310$
15

tzG mep: 5

$\begin{array}{llll}\mathrm{C} & -1.673152 & -0.512129 & -0.121922 \\ \mathrm{C} & +0.614718 & -0.509589 & +0.023993 \\ \mathrm{C} & +0.642096 & +0.951468 & -0.012427 \\ \mathrm{C} & -0.555188 & +1.683519 & -0.088860 \\ \mathrm{~S} & +3.054915 & +0.242147 & +0.146234 \\ \mathrm{O} & -0.808808 & +2.869407 & -0.122459 \\ \mathrm{~N} & -1.726772 & +0.805881 & -0.143993 \\ \mathrm{~N} & -0.531938 & -1.221902 & -0.032772 \\ \mathrm{~N} & +1.888056 & +1.503048 & +0.041845 \\ \mathrm{C} & +1.884114 & -1.064082 & +0.116568 \\ \mathrm{~N} & -2.794037 & -1.231520 & -0.183106 \\ \mathrm{H} & -2.601646 & +1.288818 & -0.200007 \\ \mathrm{H} & +2.140385 & -2.107963 & +0.155683 \\ \mathrm{H} & -3.692129 & -0.816408 & -0.301755 \\ \mathrm{H} & -2.705678 & -2.225533 & -0.200457\end{array}$

15

tzG mep: 6

$\begin{array}{llll}\text { C } & -1.673189 & -0.512286 & -0.121930\end{array}$

C $+0.615521-0.510194+0.015172$

C $+0.642055+0.951129-0.009485$

C $-0.554530+1.683064-0.094852$

$\mathrm{S}+3.054740+0.243191+0.149281$

$\begin{array}{llll}0 & -0.808714 & +2.868933 & -0.122804\end{array}$

$\begin{array}{llll}\mathrm{N} & -1.725501 & +0.805067 & -0.162778\end{array}$

N $\quad-0.531533 \quad-1.222564-0.035087$

$\mathrm{N}+1.886711+1.503319+0.059409$

C $+1.885595-1.064424+0.100378$

N $\quad-2.795121-1.230896 \quad-0.170214$

$\mathrm{H} \quad-2.600645+1.288269-0.215751$

$\mathrm{H}+2.142224-2.107826+0.148252$

H $\quad-3.698158-0.814520 \quad-0.236086$

H $\quad-2.711448 \quad-2.225116 \quad-0.140025$

15

tzG mep: 7

C -1.673384

C $\quad+0.615337$

$-0.512177$

$-0.121798$

C +0.641772

$-0.510032$

$+0.015108$

C -0.554734

$+0.951272$

$-0.007589$

S +3.054726

$+1.683246$

$-0.094770$

o $\quad-0.808335$

$+0.242580$

$+0.151132$

N $\quad-1.726187$

$+2.869125$

$-0.127315$

N $\quad-0.531617$

$+0.805567$

$-0.155580$

$\mathrm{N}+1.886854$

$-1.222240$

$-0.038974$

C +1.885266

$+1.503235$

$+0.059120$

N $\quad-2.794723$

$-1.064431$

$+0.100600$

H $\quad-2.600606$

$-1.231240$

$-0.173254$

$\mathrm{H}+2.141990$

$+1.288459$

$-0.220114$

H $\quad-3.699134$

$-2.108427$

$+0.137249$

H -2.711120

$-0.814785$

$-0.212854$

$-0.131349$ 
15

tzG mep: 8

$\begin{array}{llll}\mathrm{C} & -1.672517 & -0.512860 & -0.123633 \\ \mathrm{C} & +0.615082 & -0.509663 & +0.022853 \\ \mathrm{C} & +0.641930 & +0.951791 & -0.002975 \\ \mathrm{C} & -0.554847 & +1.683257 & -0.092851 \\ \mathrm{~S} & +3.055044 & +0.242623 & +0.144895 \\ \mathrm{O} & -0.809153 & +2.868925 & -0.127812 \\ \mathrm{~N} & -1.725465 & +0.804886 & -0.158411 \\ \mathrm{~N} & -0.531601 & -1.221894 & -0.031842 \\ \mathrm{~N} & +1.887265 & +1.503476 & +0.063389 \\ \mathrm{C} & +1.885432 & -1.064105 & +0.103476 \\ \mathrm{~N} & -2.796718 & -1.231009 & -0.167396 \\ \mathrm{H} & -2.600202 & +1.287715 & -0.222737 \\ \mathrm{H} & +2.142479 & -2.107913 & +0.143119 \\ \mathrm{H} & -3.684733 & -0.815933 & -0.349073 \\ \mathrm{H} & -2.705933 & -2.223997 & -0.219352\end{array}$

15

tzG mep: 9

C -1.672518

C +0.615084

C +0.641932

C -0.554852

$\mathrm{S}+3.055046$

O -0.809151

N -1.725461

N $\quad-0.531603$

$\mathrm{N}+1.887268$

C +1.885433

N $\quad-2.796719$

H $\quad-2.600203$

$\mathrm{H} \quad+2.142480$

H $\quad-3.684734$

H $\quad-2.705939$
$-0.512860$

$-0.509664$

$+0.951793$

$+1.683252$

$+0.242623$

$+2.868925$

$+0.804888$

$-1.221900$

$+1.503479$

$-1.064102$

$-1.231006$

$+1.287715$

$-2.107911$

$-0.815937$

$-2.223995$
$-0.123635$

$+0.022852$

$-0.002975$

$-0.092850$

$+0.144895$

$-0.127811$

$-0.158410$

$-0.031840$

$+0.063387$

$+0.103478$

$-0.167394$

$-0.222737$

$+0.143118$

$-0.349076$

$-0.219352$
13

tzI mep:0

$\begin{array}{llll}\mathrm{C} & -1.934818 & -1.439246 & -0.000073 \\ \mathrm{C} & +0.260901 & -0.946503 & +0.000024 \\ \mathrm{C} & +0.023917 & +0.465105 & -0.000014 \\ \mathrm{C} & -1.357394 & +0.981251 & +0.000178 \\ \mathrm{~S} & +2.453676 & +0.309917 & -0.000018 \\ \mathrm{O} & -1.738056 & +2.141140 & +0.000077 \\ \mathrm{~N} & -2.265739 & -0.100484 & -0.000158 \\ \mathrm{~N} & -0.726273 & -1.929243 & +0.000103 \\ \mathrm{~N} & +1.095556 & +1.287969 & -0.000118 \\ \mathrm{C} & +1.642808 & -1.190224 & +0.000045 \\ \mathrm{H} & -3.249431 & +0.166201 & -0.000092 \\ \mathrm{H} & +2.147172 & -2.158177 & +0.000124 \\ \mathrm{H} & -2.789397 & -2.125800 & -0.000100\end{array}$

13

tzI mep: 1

$\begin{array}{llll}\mathrm{C} & -1.942637 & -1.421164 & -0.000053 \\ \mathrm{C} & +0.247886 & -0.962774 & +0.000038 \\ \mathrm{C} & -0.008585 & +0.478905 & -0.000002 \\ \mathrm{C} & -1.346845 & +0.969985 & +0.000097 \\ \mathrm{~S} & +2.462876 & +0.311856 & -0.000019 \\ \mathrm{O} & -1.740179 & +2.120086 & +0.000094 \\ \mathrm{~N} & -2.273156 & -0.104394 & -0.000133 \\ \mathrm{~N} & -0.717746 & -1.897437 & +0.000081 \\ \mathrm{~N} & +1.099896 & +1.299406 & -0.000109 \\ \mathrm{C} & +1.653248 & -1.217347 & +0.000048 \\ \mathrm{H} & -3.238818 & +0.150052 & -0.000096 \\ \mathrm{H} & +2.151149 & -2.166929 & +0.000110 \\ \mathrm{H} & -2.768829 & -2.114955 & -0.000092\end{array}$

13

tzI mep:2

C $-1.951938-1.407915$

C $+0.253126-0.952912$

C $-0.007570+0.484696$

C $-1.349832+0.957159$

$\mathrm{S}+2.481214+0.307124$

○ $-1.764148+2.097842$

N $\quad-2.287222-0.104733$

N $\quad-0.720545-1.869604$

$\mathrm{N}+1.107661+1.305155$

C $+1.655788-1.228850$

H $-3.251421+0.161199$

$\mathrm{H} \quad+2.145725-2.185251$

H $\quad-2.767276-2.115985$

$-0.000018$

$+0.000030$

$-0.000008$

$-0.000002$

$-0.000015$

$+0.000081$

$-0.000077$

$+0.000034$

$-0.000055$

$+0.000045$

$-0.000046$

$+0.000094$

$-0.000040$
13

tzI mep: 3

C -1.951704

C +0.253364

$-1.407827$

$-0.000114$

C +0.253364

$-0.950905$

$-0.003202$

C -1.350876

$+0.486645$

$+0.957422$

$+0.305144$

S +2.482739

○ -1.768417

$+2.096895$

N -2.287525

$-0.104875$

N -0.719966

$-1.867943$

$\mathrm{N}+1.109391+1.305673$

C $+1.655578-1.228401$

$\mathrm{H}-3.251733+0.161789$

$\mathrm{H} \quad+2.143487 \quad-2.185987$

H $\quad-2.766772-2.116333$
$-0.009026$

$-0.000313$

$+0.005727$

$+0.006146$

$+0.002590$

$-0.001935$

$-0.012451$

$+0.004595$

$+0.007969$

$+0.009033$

$+0.000794$ 
13

tzI mep: 4

$\begin{array}{llll}\mathrm{C} & -1.951954 & -1.407729 & +0.000145 \\ \mathrm{C} & +0.253108 & -0.951107 & -0.003816 \\ \mathrm{C} & -0.007054 & +0.486529 & -0.009809 \\ \mathrm{C} & -1.350958 & +0.957514 & -0.000447 \\ \mathrm{~S} & +2.482598 & +0.305152 & +0.004898 \\ \mathrm{O} & -1.768062 & +2.097113 & +0.007240 \\ \mathrm{~N} & -2.287603 & -0.104590 & +0.001888 \\ \mathrm{~N} & -0.720286 & -1.868105 & -0.001323 \\ \mathrm{~N} & +1.109360 & +1.305509 & -0.012819 \\ \mathrm{C} & +1.655597 & -1.228490 & +0.004022 \\ \mathrm{H} & -3.251658 & +0.162370 & +0.006816 \\ \mathrm{H} & +2.143505 & -2.185999 & +0.012927 \\ \mathrm{H} & -2.767255 & -2.115915 & +0.003278\end{array}$

13

tzI mep: 5

$\begin{array}{llll}\mathrm{C} & -1.951715 & -1.407609 & -0.000601 \\ \mathrm{C} & +0.253353 & -0.950566 & +0.003891 \\ \mathrm{C} & -0.006915 & +0.486967 & +0.008220 \\ \mathrm{C} & -1.351132 & +0.957670 & +0.000443 \\ \mathrm{~S} & +2.483102 & +0.304860 & -0.003604 \\ \mathrm{O} & -1.769490 & +2.096838 & -0.005389 \\ \mathrm{~N} & -2.287762 & -0.104884 & -0.001758 \\ \mathrm{~N} & -0.720001 & -1.867650 & +0.000813 \\ \mathrm{~N} & +1.109780 & +1.305630 & +0.008012 \\ \mathrm{C} & +1.655468 & -1.228467 & -0.003506 \\ \mathrm{H} & -3.252077 & +0.161299 & -0.003264 \\ \mathrm{H} & +2.143061 & -2.186146 & -0.000563 \\ \mathrm{H} & -2.766582 & -2.116230 & -0.002548\end{array}$

13

tzI mep: 7

$\begin{array}{llll}\mathrm{C} & -1.951239 & -1.408040 & -0.002123 \\ \mathrm{C} & +0.253577 & -0.950127 & +0.003370 \\ \mathrm{C} & -0.006456 & +0.487069 & -0.001285 \\ \mathrm{C} & -1.351690 & +0.957193 & +0.001165 \\ \mathrm{~S} & +2.483178 & +0.304821 & -0.007098 \\ \mathrm{O} & -1.769839 & +2.096354 & +0.005441 \\ \mathrm{~N} & -2.287789 & -0.104971 & -0.001374 \\ \mathrm{~N} & -0.719653 & -1.867193 & +0.003194 \\ \mathrm{~N} & +1.110439 & +1.305653 & -0.003383 \\ \mathrm{C} & +1.655826 & -1.228905 & +0.005917 \\ \mathrm{H} & -3.251983 & +0.161219 & -0.005959 \\ \mathrm{H} & +2.142993 & -2.186444 & +0.007447 \\ \mathrm{H} & -2.765793 & -2.116594 & -0.004788\end{array}$

13

tzI mep: 8

C $\quad-1.951240 \quad-1.408038 \quad-0.002122$

C $+0.253574-0.950126+0.003370$

$\begin{array}{llll}\text { C } & -0.006457 & +0.487067 & -0.001287\end{array}$

C $-1.351692+0.957190+0.001166$

$\mathrm{S}+2.483180+0.304820-0.007096$

o $-1.769836+2.096354+0.005438$

N $\quad-2.287790 \quad-0.104969 \quad-0.001372$

N $\quad-0.719648-1.867195+0.003198$

$\mathrm{N} \quad+1.110439+1.305655-0.003382$

C $+1.655828-1.228900+0.005915$

$\mathrm{H} \quad-3.251985+0.161219-0.005958$

$\mathrm{H} \quad+2.142989-2.186447+0.007445$

H $\quad-2.765791-2.116594 \quad-0.004791$
13

tzI mep: 6

$\begin{array}{llll}\mathrm{C} & -1.952436 & -1.407443 & +0.000281 \\ \mathrm{C} & +0.252948 & -0.950630 & +0.004425 \\ \mathrm{C} & -0.007110 & +0.487243 & +0.009702 \\ \mathrm{C} & -1.351063 & +0.957965 & +0.001083 \\ \mathrm{~S} & +2.483575 & +0.304509 & -0.004860 \\ \mathrm{O} & -1.770276 & +2.096867 & -0.006921 \\ \mathrm{~N} & -2.287820 & -0.104718 & +0.000487 \\ \mathrm{~N} & -0.720493 & -1.867803 & -0.000115 \\ \mathrm{~N} & +1.109952 & +1.305559 & +0.007544 \\ \mathrm{C} & +1.655118 & -1.228189 & -0.003131 \\ \mathrm{H} & -3.251706 & +0.162649 & -0.001764 \\ \mathrm{H} & +2.142199 & -2.186234 & -0.008304 \\ \mathrm{H} & -2.767662 & -2.115842 & -0.003997\end{array}$




\section{LIIC Structures}

14

tzA liic:0

$\mathrm{N}+0.00000+0.00000+0.00000$

C $+1.29222+0.00000+0.00000$

$\mathrm{N}+2.20461+0.00000+1.06069$

C $+1.66218+0.01098+2.31253$

C $+0.22491+0.01220+2.45339$

C $-0.56993+0.00183+1.29210$

$\mathrm{N}-0.26115+0.01868+3.69496$

$\mathrm{S}+1.01994+0.02435+4.81238$

C $+2.27824+0.01879+3.53250$

$\mathrm{N}-1.88851+0.00331+1.31191$

$\mathrm{H}+3.32971+0.00202+3.75943$

$\mathrm{H} \quad-2.37804-0.01123+2.18194$

$\mathrm{H} \quad-2.38570-0.02801+0.44761$

$\mathrm{H} \quad+1.77300+0.00104 \quad-0.97019$

14

tzA liic: 1

$\mathrm{N}+0.00000$

C +1.29136

$+0.00000+0.00000$

$\mathrm{N}+2.18026$

$+0.00000$

$+0.00000$

C +1.59542

$+0.00000$

$+1.08610$

C +0.15022

$+0.17386$

$+2.31054$

C -0.56901

$+0.16901$

$+2.36466$

N -0.40892

$+0.03710+1.15592$

$\mathrm{S}+0.80618$

$+0.28259+3.56733$

C +2.14362

N -1.88978

$\mathrm{H}+3.18244$

$\mathrm{H} \quad-2.42788$

H $\quad-2.33842$

$+0.43169+4.75021$

$+0.30731+3.55451$

$+0.03085+1.10065$

$+0.33204+3.83675$

$+0.07548+1.94141$

$-0.06215+0.21358$

$\mathrm{H} \quad+1.78504$

$+0.01202$
14

tzA liic: 2

$\mathrm{N}+0.00000$

C +1.29050

$+0.00000$

$+0.00000$

$\mathrm{N}+2.15521$

$+0.00000$

$+0.00000$

C +1.54618

$+0.00000$

$+1.11100$

C +0.09807

$+0.33697$

$+2.29300$

C -0.56056

$+0.31628$

$+2.28388$

N -0.51431

$+0.05622$

$+1.05522$

$+0.64856$

$+0.53330$

$+3.44321$

C +2.04177

$+0.83362$

$+4.65295$

$-1.88101$

$+0.60115$

$+3.53730$

$\mathrm{H}+3.06912$

$+0.03404$

$+0.94583$

H -2.45387

$+0.67269$

$+3.85493$

$+1.75920$

$-2.29327$

$+0.13542$

$-0.12400$

$+0.04945$

H $\quad+1.79701$

$+0.02287$

$-0.95822$
14

tzA liic: 3

$\mathrm{N}+0.00000$

C +1.28964

N +2.12947

C +1.51554

C +0.06652

C -0.54969

$-0.57935$

$+0.54879$

$+1.97592$

$-1.86993$

$+2.99510$

$-2.46393$

$-2.25970$

$+1.80891$

$+0.00000$

$+0.00000$

$+0.00000$

$+0.00000$

$+0.00000$

$+0.49728$

$+1.13536$

$+0.45629$

$+2.26119$

$+0.06721$

$+2.21450$

$+0.77237$

$+0.99166$

$+1.22368$

$+3.32953$

$+0.89245$

$+4.52853$

$+0.02573$

$+3.48326$

$+1.01209$

$+0.85081$

$+0.18180$

$+3.81563$

$-0.19751$

$+1.64103$

$+0.03357$

0.04266

$-0.95180$

14

tzA liic: 4

$\mathrm{N}+0.00000$

C +1.28878

$+0.00000+0.00000$

$\mathrm{N}+2.10306$

$+0.00000$

$+0.00000$

C +1.50416

$+0.00000$

$+1.15916$

C +0.05413

$+0.65177$

$+2.21667$

C -0.53995

$+0.59176$

$+2.15875$

N -0.60568

$+0.07841$

$+0.96487$

S +0.50755

$+1.00230$

$+3.23143$

$+1.59640$

$+4.38461$

C +1.94811

$+1.17337$

$+3.39579$

N -1.86185

$+0.01944$

$+0.81566$

$\mathrm{H} \quad+2.96382$

$+1.33816$

$+3.72246$

H -2.46353

$+0.22878$

$+1.58859$

H $\quad-2.24417$

$-0.26596$

$-0.06413$

$\mathrm{H} \quad+1.82073$

$+0.04412$

$-0.94508$

14

tzA liic: 5

$\mathrm{N}+0.00000$

C +1.28792

$+0.00000$

$+0.00000$

$+0.00000+0.00000$

N +2.07598

$+0.00000$

$+1.18240$

C +1.51226

$+0.79749$

$+2.16128$

C +0.06022

$+0.72540$

$+2.11772$

C $\quad-0.53317$

$+0.09776$

$+0.97261$

N -0.59419

$+1.22587$

$+3.15214$

$S \quad+0.52480$

$+1.94700$

$+4.22823$

C +1.95909

$+1.43632$

$+3.27914$

N -1.85931

$+0.02831$

$+0.83743$

$\mathrm{H} \quad+2.97666$

$+1.63917+3.58078$

H -2.45516

$+0.29009+1.59986$

H $\quad-2.24972$

$-0.31307-0.01950$

$\mathrm{H}+1.83248+0.05451-0.93808$ 
14

\begin{tabular}{llll}
\multicolumn{1}{l}{$t z A$ lic: 6} & \\
$N$ & +0.00000 & +0.00000 & +0.00000 \\
$C$ & +1.28706 & +0.00000 & +0.00000 \\
$N$ & +2.04826 & +0.00000 & +1.20504 \\
$C$ & +1.53963 & +0.93158 & +2.09701 \\
$C$ & +0.08487 & +0.85951 & +2.09139 \\
$C$ & -0.52933 & +0.13233 & +1.01120 \\
$N$ & -0.54481 & +1.44527 & +3.09307 \\
$S$ & +0.59986 & +2.27113 & +4.06564 \\
$C$ & +2.00832 & +1.67423 & +3.13820 \\
$N$ & -1.86184 & +0.06411 & +0.91067 \\
$H$ & +3.03280 & +1.90426 & +3.39738 \\
$H$ & -2.43810 & +0.37784 & +1.66960 \\
$H$ & -2.27575 & -0.32396 & +0.08424 \\
$H$ & +1.84415 & +0.06474 & -0.93080
\end{tabular}

14

tzA liic: 7

$\begin{array}{llll}\mathrm{N} & +0.00000 & +0.00000 & +0.00000 \\ \mathrm{C} & +1.28620 & +0.00000 & +0.00000 \\ \mathrm{~N} & +2.01989 & +0.00000 & +1.22707 \\ \mathrm{C} & +1.58556 & +1.05138 & +2.02603 \\ \mathrm{C} & +0.12898 & +0.99552 & +2.07893 \\ \mathrm{C} & -0.52670 & +0.18770 & +1.07595 \\ \mathrm{~N} & -0.45658 & +1.66148 & +3.05402 \\ \mathrm{~S} & +0.73159 & +2.56437 & +3.90216 \\ \mathrm{C} & +2.09397 & +1.88072 & +2.97834 \\ \mathrm{~N} & -1.86611 & +0.13632 & +1.02809 \\ \mathrm{H} & +3.12925 & +2.12397 & +3.18011 \\ \mathrm{H} & -2.40860 & +0.50120 & +1.79011 \\ \mathrm{H} & -2.31811 & -0.28611 & +0.23830 \\ \mathrm{H} & +1.85574 & +0.07479 & -0.92325\end{array}$

14

tzA liic: 8

$\begin{array}{llll}\mathrm{N} & +0.00000 & +0.00000 & +0.00000 \\ \mathrm{C} & +1.28534 & +0.00000 & +0.00000 \\ \mathrm{~N} & +1.99090 & +0.00000 & +1.24848 \\ \mathrm{C} & +1.64890 & +1.15445 & +1.95055 \\ \mathrm{C} & +0.19402 & +1.13363 & +2.07887 \\ \mathrm{C} & -0.52205 & +0.26765 & +1.16157 \\ \mathrm{~N} & -0.32788 & +1.87358 & +3.03362 \\ \mathrm{~S} & +0.91826 & +2.82195 & +3.74223 \\ \mathrm{C} & +2.21299 & +2.05030 & +2.80515 \\ \mathrm{~N} & -1.86628 & +0.25137 & +1.18129 \\ \mathrm{H} & +3.26106 & +2.29069 & +2.93745 \\ \mathrm{H} & -2.36038 & +0.66551 & +1.95206 \\ \mathrm{H} & -2.36941 & -0.19008 & +0.43288 \\ \mathrm{H} & +1.86725 & +0.08467 & -0.91542\end{array}$

14

$\begin{array}{llll}\text { tzA liic: } & & & \\ \mathrm{N} & +0.00000 & +0.00000 & +0.00000 \\ \mathrm{C} & +1.28448 & +0.00000 & +0.00000 \\ \mathrm{~N} & +1.96131 & +0.00000 & +1.26924 \\ \mathrm{C} & +1.72805 & +1.23861 & +1.87284 \\ \mathrm{C} & +0.28182 & +1.27261 & +2.08943 \\ \mathrm{C} & -0.51103 & +0.37388 & +1.26268 \\ \mathrm{~N} & -0.15687 & +2.07830 & +3.02970 \\ \mathrm{~S} & +1.15741 & +3.03842 & +3.58948 \\ \mathrm{C} & +2.36122 & +2.17853 & +2.62424 \\ \mathrm{~N} & -1.85455 & +0.41212 & +1.36146 \\ \mathrm{H} & +3.42157 & +2.39892 & +2.67810 \\ \mathrm{H} & -2.28539 & +0.87158 & +2.14544 \\ \mathrm{H} & -2.41949 & -0.03018 & +0.65792 \\ \mathrm{H} & +1.87868 & +0.09436 & -0.90732\end{array}$

15

tzG liic:0

$\begin{array}{llll}\mathbf{N} & +0.00000 & +0.00000 & +0.00000 \\ \mathrm{C} & +1.31927 & +0.00000 & +0.00000 \\ \mathrm{~N} & +2.07569 & +0.00000 & +1.11385 \\ \mathrm{C} & +1.41175 & +0.00371 & +2.29042 \\ \mathrm{C} & -0.04762 & -0.00340 & +2.37656 \\ \mathrm{C} & -0.82865 & -0.00950 & +1.20807 \\ \mathrm{~N} & -0.54694 & -0.00106 & +3.64553 \\ \mathrm{~S} & +0.76147 & +0.02403 & +4.76246 \\ \mathrm{C} & +2.01866 & +0.01967 & +3.53906 \\ \mathrm{~N} & +1.99032 & -0.01300 & -1.15369 \\ \mathrm{O} & -2.02408 & -0.01938 & +1.00178 \\ \mathrm{H} & -0.51907 & -0.00459 & -0.85613 \\ \mathrm{H} & +3.07263 & +0.02170 & +3.75431 \\ \mathrm{H} & +1.53529 & +0.10021 & -2.03347 \\ \mathrm{H} & +2.98443 & +0.06831 & -1.10800\end{array}$

15

tzG liic: 1

$\begin{array}{llll}\mathrm{N} & +0.00000 & +0.00000 & +0.00000 \\ \mathrm{C} & +1.32797 & +0.00000 & +0.00000 \\ \mathrm{~N} & +2.05932 & +0.00000 & +1.13444 \\ \mathrm{C} & +1.36186 & +0.15214 & +2.28283 \\ \mathrm{C} & -0.09820 & +0.22202 & +2.30662 \\ \mathrm{C} & -0.83696 & +0.12679 & +1.10338 \\ \mathrm{~N} & -0.64646 & +0.36453 & +3.53864 \\ \mathrm{~S} & +0.60909 & +0.43108 & +4.70774 \\ \mathrm{C} & +1.91376 & +0.24851 & +3.55378 \\ \mathrm{~N} & +1.99185 & +0.09812 & -1.16544 \\ \mathrm{O} & -2.02512 & +0.13874 & +0.86696 \\ \mathrm{H} & -0.51942 & -0.05071 & -0.85607 \\ \mathrm{H} & +2.95598 & +0.21783 & +3.81689 \\ \mathrm{H} & +1.50207 & +0.20815 & -2.02964 \\ \mathrm{H} & +2.99028 & +0.13948 & -1.11500\end{array}$


15

tzG liic: 2

$\begin{array}{llll}\mathrm{N} & +0.00000 & +0.00000 & +0.00000 \\ \mathrm{C} & +1.33667 & +0.00000 & +0.00000 \\ \mathrm{~N} & +2.04239 & +0.00000 & +1.15447 \\ \mathrm{C} & +1.32802 & +0.30013 & +2.26409 \\ \mathrm{C} & -0.12644 & +0.44313 & +2.22825 \\ \mathrm{C} & -0.83510 & +0.25437 & +1.00670 \\ \mathrm{~N} & -0.70651 & +0.72422 & +3.41255 \\ \mathrm{~S} & +0.50430 & +0.83831 & +4.61926 \\ \mathrm{C} & +1.83624 & +0.48157 & +3.54451 \\ \mathrm{~N} & +1.99312 & +0.21133 & -1.16634 \\ \mathrm{O} & -2.01644 & +0.28886 & +0.74783 \\ \mathrm{H} & -0.51977 & -0.09684 & -0.85352 \\ \mathrm{H} & +2.86481 & +0.42220 & +3.85134 \\ \mathrm{H} & +1.47667 & +0.31321 & -2.01880 \\ \mathrm{H} & +2.99466 & +0.21248 & -1.11914\end{array}$

15

tzG liic: 3

$\begin{array}{llll}\mathrm{N} & +0.00000 & +0.00000 & +0.00000 \\ \mathrm{C} & +1.34538 & +0.00000 & +0.00000 \\ \mathrm{~N} & +2.02491 & +0.00000 & +1.17392 \\ \mathrm{C} & +1.31088 & +0.44538 & +2.23538 \\ \mathrm{C} & -0.13251 & +0.65834 & +2.14350 \\ \mathrm{C} & -0.82493 & +0.37521 & +0.91958 \\ \mathrm{~N} & -0.72701 & +1.07413 & +3.27096 \\ \mathrm{~S} & +0.44892 & +1.23869 & +4.50097 \\ \mathrm{C} & +1.78815 & +0.71380 & +3.51301 \\ \mathrm{~N} & +1.99414 & +0.32557 & -1.15611 \\ \mathrm{O} & -2.00022 & +0.43467 & +0.64573 \\ \mathrm{H} & -0.52011 & -0.14284 & -0.84848 \\ \mathrm{H} & +2.80204 & +0.62881 & +3.85891 \\ \mathrm{H} & +1.45956 & +0.41562 & -2.00162 \\ \mathrm{H} & +2.99750 & +0.28754 & -1.12011\end{array}$

15

tzG liic: 4

$\begin{array}{llll}\mathrm{N} & +0.00000 & +0.00000 & +0.00000 \\ \mathrm{C} & +1.35408 & +0.00000 & +0.00000 \\ \mathrm{~N} & +2.00691 & +0.00000 & +1.19278 \\ \mathrm{C} & +1.31077 & +0.58562 & +2.19806 \\ \mathrm{C} & -0.11673 & +0.86625 & +2.05437 \\ \mathrm{C} & -0.80795 & +0.49157 & +0.84309 \\ \mathrm{~N} & -0.70837 & +1.41069 & +3.11762 \\ \mathrm{~S} & +0.44376 & +1.62539 & +4.35736 \\ \mathrm{C} & +1.77080 & +0.94007 & +3.46155 \\ \mathrm{~N} & +1.99489 & +0.43974 & -1.13455 \\ \mathrm{O} & -1.97785 & +0.58004 & +0.56130 \\ \mathrm{H} & -0.52045 & -0.18857 & -0.84096 \\ \mathrm{H} & +2.76973 & +0.83145 & +3.84157 \\ \mathrm{H} & +1.45116 & +0.51578 & -1.97855 \\ \mathrm{H} & +2.99877 & +0.36481 & -1.11747\end{array}$

15

\begin{tabular}{llll}
\multicolumn{4}{c}{ tzG liic:5 } \\
$\mathrm{N}$ & +0.00000 & +0.00000 & +0.00000 \\
$\mathrm{C}$ & +1.36279 & +0.00000 & +0.00000 \\
$\mathrm{~N}$ & +1.98840 & +0.00000 & +1.21103 \\
$\mathrm{C}$ & +1.32769 & +0.71861 & +2.15361 \\
$\mathrm{C}$ & -0.07956 & +1.06554 & +1.96276 \\
$\mathrm{C}$ & -0.78517 & +0.60582 & +0.77782 \\
$\mathrm{~N}$ & -0.65151 & +1.73067 & +2.95629 \\
$\mathrm{~S}$ & +0.48857 & +1.99189 & +4.19326 \\
$\mathrm{C}$ & +1.78475 & +1.15535 & +3.39277 \\
$\mathrm{~N}$ & +1.99539 & +0.55268 & -1.10159 \\
$\mathrm{O}$ & -1.94988 & +0.72882 & +0.49451 \\
$\mathrm{H}$ & -0.52080 & -0.23391 & -0.83097 \\
$\mathrm{H}$ & +2.76896 & +1.02391 & +3.80187 \\
$\mathrm{H}$ & +1.45173 & +0.61418 & -1.94978 \\
$\mathrm{H}$ & +2.99842 & +0.44440 & -1.11075
\end{tabular}

15

tzG liic: 6

$\begin{array}{llll}\mathrm{N} & +0.00000 & +0.00000 & +0.00000 \\ \mathrm{C} & +1.37149 & +0.00000 & +0.00000 \\ \mathrm{~N} & +1.96939 & +0.00000 & +1.22866 \\ \mathrm{C} & +1.36130 & +0.84221 & +2.10363 \\ \mathrm{C} & -0.02158 & +1.25505 & +1.87055 \\ \mathrm{C} & -0.75707 & +0.72033 & +0.72399 \\ \mathrm{~N} & -0.55773 & +2.03114 & +2.79065 \\ \mathrm{~S} & +0.58211 & +2.33214 & +4.01374 \\ \mathrm{C} & +1.82972 & +1.35475 & +3.30968 \\ \mathrm{~N} & +1.99563 & +0.66326 & -1.05728 \\ \mathrm{O} & -1.91598 & +0.88460 & +0.44481 \\ \mathrm{H} & -0.52114 & -0.27872 & -0.81854 \\ \mathrm{H} & +2.79977 & +1.20013 & +3.74283 \\ \mathrm{H} & +1.46144 & +0.71130 & -1.91526 \\ \mathrm{H} & +2.99640 & +0.52630 & -1.09937\end{array}$

15

tzG 1iic: 7

$\mathrm{N}+0.00000+0.00000+0.00000$

C $+1.38019+0.00000+0.00000$

$\mathrm{N}+1.94989+0.00000+1.24565$

C $+1.41094+0.95441+2.04974$

C $+0.05654+1.43365+1.77947$

C $-0.72354+0.83729+0.68146$

$\mathrm{N}-0.42872+2.30946+2.62428$

$\mathrm{S}+0.72217+2.64059+3.82398$

C $+1.90464+1.53367+3.21545$

$\mathrm{N}+1.99561+0.77032-1.00176$

O $\quad-1.87490+1.05044+0.41127$

$\mathrm{H} \quad-0.52148 \quad-0.32286-0.80369$

$\mathrm{H}+2.86116+1.35434+3.66784$

$\mathrm{H} \quad+1.48033+0.80760-1.87465$

$\mathrm{H} \quad+2.99269+0.61042 \quad-1.08273$ 
15

tzG liic: 8

$\begin{array}{llll}\mathrm{N} & +0.00000 & +0.00000 & +0.00000 \\ \mathrm{C} & +1.38890 & +0.00000 & +0.00000 \\ \mathrm{~N} & +1.92991 & +0.00000 & +1.26200 \\ \mathrm{C} & +1.47559 & +1.05335 & +1.99359 \\ \mathrm{C} & +0.15404 & +1.60028 & +1.69119 \\ \mathrm{C} & -0.68393 & +0.95858 & +0.64987 \\ \mathrm{~N} & -0.26652 & +2.56329 & +2.46056 \\ \mathrm{~S} & +0.90563 & +2.91230 & +3.62910 \\ \mathrm{C} & +2.00762 & +1.68790 & +3.11340 \\ \mathrm{~N} & +1.99533 & +0.87272 & -0.93532 \\ \mathrm{O} & -1.82456 & +1.22871 & +0.39269 \\ \mathrm{H} & -0.52181 & -0.36622 & -0.78645 \\ \mathrm{H} & +2.95106 & +1.48126 & +3.58050 \\ \mathrm{H} & +1.50832 & +0.90340 & -1.82737 \\ \mathrm{H} & +2.98724 & +0.69655 & -1.06018\end{array}$

15

tzG liic: 9

$\begin{array}{llll}\mathrm{N} & +0.00000 & +0.00000 & +0.00000 \\ \mathrm{C} & +1.39760 & +0.00000 & +0.00000 \\ \mathrm{~N} & +1.90949 & +0.00000 & +1.27768 \\ \mathrm{C} & +1.55396 & +1.13737 & +1.93681 \\ \mathrm{C} & +0.27005 & +1.75386 & +1.60725 \\ \mathrm{C} & -0.63707 & +1.08561 & +0.62869 \\ \mathrm{~N} & -0.07344 & +2.79053 & +2.30269 \\ \mathrm{~S} & +1.12853 & +3.14297 & +3.43407 \\ \mathrm{C} & +2.13601 & +1.81368 & +3.00685 \\ \mathrm{~N} & +1.99479 & +0.96932 & -0.85835 \\ \mathrm{O} & -1.76216 & +1.42083 & +0.38781 \\ \mathrm{H} & -0.52215 & -0.40866 & -0.76688 \\ \mathrm{H} & +3.06641 & +1.57621 & +3.48450 \\ \mathrm{H} & +1.54518 & +0.99881 & -1.77263 \\ \mathrm{H} & +2.98003 & +0.78434 & -1.03107\end{array}$

13

tzI liic: 0

$\begin{array}{llll}\mathrm{N} & +0.00000 & +0.00000 & +0.00000 \\ \mathrm{C} & +1.34583 & +0.00000 & +0.00000 \\ \mathrm{~N} & +2.09837 & +0.00000 & +1.07765 \\ \mathrm{C} & +1.45382 & +0.00642 & +2.24927 \\ \mathrm{C} & -0.00274 & +0.01246 & +2.35687 \\ \mathrm{C} & -0.79433 & +0.00414 & +1.17197 \\ \mathrm{~N} & -0.51601 & +0.02169 & +3.64295 \\ \mathrm{~S} & +0.79630 & +0.03041 & +4.72177 \\ \mathrm{C} & +2.07440 & +0.01038 & +3.53725 \\ \mathrm{O} & -2.00186 & -0.00010 & +1.05199 \\ \mathrm{H} & -0.49885 & +0.00025 & -0.86701 \\ \mathrm{H} & +3.12334 & +0.00950 & +3.76950 \\ \mathrm{H} & +1.82818 & -0.00274 & -0.96586\end{array}$

13

\begin{tabular}{llll}
\multicolumn{3}{l}{ tzI liic: 1} \\
$\mathrm{~N}$ & +0.00000 & +0.00000 & +0.00000 \\
$\mathrm{C}$ & +1.35224 & +0.00000 & +0.00000 \\
$\mathrm{~N}$ & +2.08070 & +0.00000 & +1.10158 \\
$\mathrm{C}$ & +1.40165 & +0.17187 & +2.24690 \\
$\mathrm{C}$ & -0.05617 & +0.24991 & +2.27773 \\
$\mathrm{C}$ & -0.77134 & +0.13646 & +1.17878 \\
$\mathrm{~N}$ & -0.62741 & +0.41257 & +3.51969 \\
$\mathrm{~S}$ & +0.62496 & +0.48193 & +4.66242 \\
$\mathrm{C}$ & +1.95493 & +0.27877 & +3.55426 \\
$\mathrm{O}$ & -1.98035 & +0.15945 & +1.08818 \\
$\mathrm{H}$ & -0.50168 & -0.03148 & -0.86642 \\
$\mathrm{H}$ & +2.98918 & +0.25760 & +3.84503 \\
$\mathrm{H}$ & +1.84080 & +0.06043 & -0.96302
\end{tabular}

13

tzI liic: 2

$\mathrm{N}+0.00000+0.00000+0.00000$

C $+1.35865+0.00000+0.00000$

$\mathrm{N}+2.06230+0.00000+1.12498$

C $+1.36909+0.33708+2.23066$

C $-0.08327+0.48023+2.19156$

C $-0.74808+0.27023+1.17008$

N $-0.69122+0.79331+3.37706$

$\mathrm{S}+0.51255+0.93183+4.56190$

C $+1.87144+0.55168+3.53876$

o $-1.95660+0.32621+1.10169$

H $\quad-0.50453 \quad-0.06316-0.86467$

$\mathrm{H}+2.89011+0.51663+3.87964$

$\mathrm{H}+1.85343+0.12322-0.95601$

13

tzI liic: 3

$\mathrm{N}+0.00000+0.00000+0.00000$

C $+1.36506+0.00000+0.00000$

$\mathrm{N}+2.04317+0.00000+1.14783$

C $+1.35705+0.49883+2.20208$

C $-0.08483+0.70197+2.10129$

C $-0.72455+0.40357+1.14565$

$\mathrm{N}-0.70826+1.15957+3.22062$

$\mathrm{S}+0.46094+1.37060+4.42625$

C $+1.82654+0.82145+3.49354$

o $\quad-1.93061+0.49701+1.09137$

$\mathrm{H} \quad-0.50737 \quad-0.09475-0.86175$

$\mathrm{H} \quad+2.83043+0.77693+3.87537$

$\mathrm{H}+1.86607+0.18535-0.94488$ 
13

\begin{tabular}{llll}
\multicolumn{3}{l}{$t z I$ lic: 4} \\
$N$ & +0.00000 & +0.00000 & +0.00000 \\
$\mathrm{C}$ & +1.37147 & +0.00000 & +0.00000 \\
$\mathrm{~N}$ & +2.02334 & +0.00000 & +1.17010 \\
$\mathrm{C}$ & +1.36598 & +0.65391 & +2.16292 \\
$\mathrm{C}$ & -0.06174 & +0.91400 & +2.00961 \\
$\mathrm{C}$ & -0.70078 & +0.53458 & +1.10550 \\
$\mathrm{~N}$ & -0.67995 & +1.50769 & +3.05575 \\
$\mathrm{~S}$ & +0.47045 & +1.78924 & +4.26216 \\
$\mathrm{C}$ & +1.82159 & +1.08047 & +3.42219 \\
$\mathrm{O}$ & -1.90237 & +0.66851 & +1.05653 \\
$\mathrm{H}$ & -0.51022 & -0.12621 & -0.85766 \\
$\mathrm{H}$ & +2.81281 & +1.02864 & +3.83551 \\
$\mathrm{H}$ & +1.87870 & +0.24653 & -0.92968
\end{tabular}

13

tzI liic: 5

$\begin{array}{llll}\mathrm{N} & +0.00000 & +0.00000 & +0.00000 \\ \mathrm{C} & +1.37788 & +0.00000 & +0.00000 \\ \mathrm{~N} & +2.00281 & +0.00000 & +1.19177 \\ \mathrm{C} & +1.39580 & +0.79917 & +2.11521 \\ \mathrm{C} & -0.01491 & +1.11543 & +1.91896 \\ \mathrm{C} & -0.67676 & +0.66134 & +1.04988 \\ \mathrm{~N} & -0.60812 & +1.83460 & +2.88754 \\ \mathrm{~S} & +0.53991 & +2.17954 & +4.07672 \\ \mathrm{C} & +1.85665 & +1.32144 & +3.32894 \\ \mathrm{O} & -1.87187 & +0.83727 & +0.99694 \\ \mathrm{H} & -0.51307 & -0.15750 & -0.85242 \\ \mathrm{H} & +2.83816 & +1.26207 & +3.76439 \\ \mathrm{H} & +1.89135 & +0.30651 & -0.91049\end{array}$

13

tzI liic: 6

$\begin{array}{llll}\mathrm{N} & +0.00000 & +0.00000 & +0.00000 \\ \mathrm{C} & +1.38429 & +0.00000 & +0.00000 \\ \mathrm{~N} & +1.98161 & +0.00000 & +1.21283 \\ \mathrm{C} & +1.44593 & +0.93163 & +2.06107 \\ \mathrm{C} & +0.05477 & +1.30543 & +1.83152 \\ \mathrm{C} & -0.65250 & +0.78199 & +0.97927 \\ \mathrm{~N} & -0.49493 & +2.13772 & +2.72073 \\ \mathrm{~S} & +0.66681 & +2.53408 & +3.87719 \\ \mathrm{C} & +1.93046 & +1.53750 & +3.21844 \\ \mathrm{O} & -1.83913 & +0.99982 & +0.91284 \\ \mathrm{H} & -0.51592 & -0.18857 & -0.84603 \\ \mathrm{H} & +2.90555 & +1.46804 & +3.66713 \\ \mathrm{H} & +1.90399 & +0.36502 & -0.88741\end{array}$

13

\begin{tabular}{llll}
\multicolumn{3}{l}{$t z I$ lic: 7} \\
$N \quad+0.00000$ & +0.00000 & +0.00000 \\
$\mathrm{C}$ & +1.39070 & +0.00000 & +0.00000 \\
$\mathrm{~N}$ & +1.95973 & +0.00000 & +1.23325 \\
$\mathrm{C}$ & +1.51526 & +1.04847 & +2.00273 \\
$\mathrm{C}$ & +0.14648 & +1.48321 & +1.74919 \\
$\mathrm{C}$ & -0.62802 & +0.89471 & +0.89440 \\
$\mathrm{~N}$ & -0.34278 & +2.41479 & +2.55965 \\
$\mathrm{~S}$ & +0.84736 & +2.84639 & +3.67073 \\
$\mathrm{C}$ & +2.04045 & +1.72250 & +3.09560 \\
$\mathrm{O}$ & -1.80416 & +1.15269 & +0.80498 \\
$\mathrm{H}$ & -0.51878 & -0.21939 & -0.83850 \\
$\mathrm{H}$ & +3.01226 & +1.63820 & +3.54935 \\
$\mathrm{H}$ & +1.91664 & +0.42181 & -0.86054
\end{tabular}

13

tzI liic: 8

$\begin{array}{llll}\mathrm{N} & +0.00000 & +0.00000 & +0.00000 \\ \mathrm{C} & +1.39711 & +0.00000 & +0.00000 \\ \mathrm{~N} & +1.93720 & +0.00000 & +1.25301 \\ \mathrm{C} & +1.60218 & +1.14718 & +1.94243 \\ \mathrm{C} & +0.25940 & +1.64784 & +1.67370 \\ \mathrm{C} & -0.60333 & +0.99778 & +0.79620 \\ \mathrm{~N} & -0.15422 & +2.66376 & +2.40818 \\ \mathrm{~S} & +1.07665 & +3.11093 & +3.46424 \\ \mathrm{C} & +2.18288 & +1.87113 & +2.96538 \\ \mathrm{O} & -1.76698 & +1.29253 & +0.67459 \\ \mathrm{H} & -0.52164 & -0.24990 & -0.82984 \\ \mathrm{H} & +3.15385 & +1.76536 & +3.41694 \\ \mathrm{H} & +1.92929 & +0.47663 & -0.83001\end{array}$

13

tzI liic: 9

$\begin{array}{llll}\mathrm{N} & +0.00000 & +0.00000 & +0.00000 \\ \mathrm{C} & +1.40353 & +0.00000 & +0.00000 \\ \mathrm{~N} & +1.91404 & +0.00000 & +1.27209 \\ \mathrm{C} & +1.70461 & +1.22555 & +1.88236 \\ \mathrm{C} & +0.39272 & +1.79818 & +1.60657 \\ \mathrm{C} & -0.57842 & +1.08959 & +0.68583 \\ \mathrm{~N} & +0.06801 & +2.88264 & +2.26981 \\ \mathrm{~S} & +1.34882 & +3.32313 & +3.26420 \\ \mathrm{C} & +2.35294 & +1.97903 & +2.83260 \\ \mathrm{O} & -1.72761 & +1.41616 & +0.52336 \\ \mathrm{H} & -0.52450 & -0.28007 & -0.82006 \\ \mathrm{H} & +3.32433 & +1.84365 & +3.27576 \\ \mathrm{H} & +1.94194 & +0.52924 & -0.79597\end{array}$

